\title{
DIVISION II: THE SUN AND HELIOSPHERE
}

\section{(SOLEIL ET HELIOSPHERE)}

\author{
PRESIDENT: Arnold O. Benz
}

BOARD: Thomas J. Bogdan, Peter V. Foukal, Donald B. Melrose, Sami K. Solanki, Marek Vandas \& David F. Webb

\author{
Commission 10: Solar Activity \\ Commission 12: Solar Radiation and Structure \\ Commission 49: Interplanetary Plasma and Heliosphere
}

\section{Introduction}

Division II consists of Commissions 10 (Solar Activity), 12 (Solar Radiation and Structure), and 49 (Interplanetary Plasma and Heliosphere). Detailed information on IAU activities in these three areas can be found in the individual reports of each Commission.

The Division II Board was active in transferring information to the members of its Commissions, setting up a web page, corresponding with the Working Groups, initiating a new one, evaluating proposed IAU conferences, and propose conferences to be cosponsored.

\section{Scientific Highlights}

Highlights of scientific progress are contained in the three Commission reports. Commission 10 concentrates on the short term dynamics of solar variability and magnetic activity, noting in particular the advances in coronal seismology in EUV lines, continuous coronal variability indicating substantial mass and energy input in quiet regions suggestive for coronal heating, and the solar origin of coronal mass ejections (CME). Commission 12 encompasses the solar interior, atmospheric structure, and irradiance. It reports on new findings in global and local seismology, chromospheric oscillations, structure of coronal loops, and surface magnetism. Commission 49 concentrates on interplanetary space, solar wind dynamics and termination shock. Its report includes discoveries of transient interplanetary shocks, unusual composition of ions in relation to CMEs, physics of solar system dusty plasmas, remote sensing of the inner heliosphere, and new models for CMEs and the outer heliosphere. As all Commissions point out, the reports cannot be considered complete in view of the many hundred publications in the period.

\section{Special Projects and Other Activities}

Special activities supported in part by our Division include the Quarterly Bulletin of Solar Activity (QBSA), the Sunspot Index Data Center, and the Deberecen Photoheliography Data (DPD).

In particular, we thank Dr. K. Shibasaki for his work on the QBSA. Also, we are grateful to Dr. P. Cugnon of SID for the excellent work in that data center, now accessible at http://sidc.oma.be/index.php3. We thank Dr. A. Ludmany (ludmany@tigris.klte.hu) and his colleagues at Debrecen for producing daily full-disk photoheliograms.

\section{Workshops, Colloquia and Conferences}

Several major conferences relating to the scope of Division II were held, of which the following may be mentioned: 
IAU Symposium 203 on 'Recent Insight into the Physics of the Sun and Heliosphere: highlights from SOHO and other space missions', Manchester, UK, August 7-11, 2000

'The Physics of the Solar Corona and Transition Region', Monterey, California, 24-27 August, 1999

International Conference on 'Global Picture of Solar Eruptive Events', Washington DC, March 6-9, 2000

CESRA Workshop on 'Energy Conversion and Particle Acceleration in the Solar Corona', Tegernsee, Germany, July 2-6, 2001

'Multi-Wavelength Observations of Coronal Structure and Dynamics', Yohkoh 10th anniversary, Hawaii, January 21-24, 2002

'Sunspots and Starspots', Potsdam Thinkshop, Potsdam, May 6-10, 2002 [http://www.aip. de./thinkshop/]

IAU Colloquium 188 on 'Magnetic Coupling of the Solar Atmosphere', Santorin, Greece, June 11-15, 2002

European Solar Physics Meeting 'Solar Variability: from Core to Outer Frontiers', Prague, September 9-14, 2002

\section{Major Publications}

Important publications in the period of this report are listed in the Commission Reports.

\section{Working Groups}

The reports of the Working Group on Solar Eclipses and the Working Group on Solar and Interplanetary Nomenclature are printed separately.

A Working Group for Solar Data Distribution has been initiated to study the archiving, retrieval and distribution of solar data. The immediate reason are considerations on the future of the Quarterly Bulletin of Solar Activity (QBSO), which has been supported by the IAU for many years. The need for a review of existing means of data distribution is obvious in view of the revolution in digital data storage and communication.

The intent of the Working Group is to survey the existing and growing data exchange through the Internet and to propose guidelines at an international level. The existing structures at the national levels should not be replaced nor duplicated.

The Working Group studies electronic means to support

- Finding characteristic parameters of solar activity, solar events and active regions (lists similar to today's QBSO and Solar Geophysical Data (SGD)).

- Finding available data from the various observatories and satellites.

- Finding quick-look data (such as in today's SOHO data center, Base2000 etc.)

- Finding actual raw data or calibrated data in archives located around the world.

- Downloading software for the data analysis

The goal is to facilitate the use of the available solar data that is archived in a large number of computers scattered all over the world.

\section{Sources of Further Information}

Information on upcoming Division II events and updates on Commission activities and Working Groups are posted on the Division and Commission web pages on the IAU site at http://www.iau.org. 